\title{
Les deux langues en clair-obscur dans Mimodrames et Icônes de Juan Mari Lekuona
}

\section{Aurelia Arcocha-Scarcia}

\section{(2) OpenEdition}

1 Journals

\section{Édition électronique}

URL : http://journals.openedition.org/genesis/2662

DOI : 10.4000/genesis.2662

ISSN : 2268-1590

\section{Éditeur :}

Presses universitaires de Paris Sorbonne (PUPS), Société internationale de génétique artistique littéraire et scientifique (SIGALES)

\section{Édition imprimée}

Date de publication : 4 juin 2018

Pagination : 65-80

ISBN : 979-10-231-0604-6

ISSN : 1167-5101

\section{Référence électronique}

Aurelia Arcocha-Scarcia, « Les deux langues en clair-obscur dans Mimodrames et Icônes de Juan Mari Lekuona », Genesis [En ligne], 46 | 2018, mis en ligne le 01 juin 2019, consulté le 07 septembre 2019 URL : http://journals.openedition.org/genesis/2662 ; DOI : 10.4000/genesis.2662 


\title{
Les deux langues en clair-obscur dans Mimodrames et Icônes de Juan Mari Lekuona*
}

\author{
Aurelia Arcocha-Scarcia
}

... nero, bianco, benchè questi non sono messi fra' colori, perchè l'uno
è tenebre, l'altro è luce, cioè l'uno è privazione e l'altro è generativo :
mà io non gli voglio per questo lasciare in dietro, perchè in pittura
sono li principali, conciosiachè la pittura sia composta d'ombre,
e di lumi, ciò̀ di chiaro, e d'oscuro**.

Leonardo DA VINCI

\section{Introduction}

Juan Mari Lekuona est un poète, universitaire et traducteur ${ }^{1}$ d'expression basque né le 11 novembre 1927 à Oyarzun et mort le 5 décembre 2005 à Saint-Sébastien, en Espagne. Il publie Mimodrames et Icônes, l'œuvre sur laquelle portera notre analyse, en 1990 à Saint-Sébastien.

Pour comprendre les enjeux qui se présentent à un auteur bilingue qui écrit en langue basque, il est nécessaire d'évoquer rapidement la situation des langues de l'Espagne depuis la fin du franquisme (1975).

Comme pour le catalan et le galicien dans leurs territoires autonomes respectifs, la langue basque - ou euskara - se voit dotée d'un statut de co-officialité à partir de 1982.
Diverses aides du gouvernement autonome basque ainsi que des politiques culturelles volontaristes encouragent l'émergence d'un champ littéraire basque contemporain (publications, traductions, prix, etc.); parallèlement surgissent aussi de nouvelles universités publiques ${ }^{2}$ et des instances médiatiques.

Dans un tel contexte de bilinguisme officiel, l'autotraduction se trouve naturellement favorisée. Déjà en 2002, Julio César Santoyo faisait remarquer que «jamais la péninsule Ibérique n'avait connu une telle prolifération de traductions d'auteurs comme en cette fin de $\mathrm{XX}^{\mathrm{e}}$ siècle ${ }^{3}$ » et qu'il y avait réellement un corpus «immense, toujours plus vaste, de textes traduits par leurs propres créateurs ${ }^{4} »$. Les analyses de Rainier Grutman ${ }^{5}$, de Christian Lagarde ${ }^{6}$, de Nayrouz

(*) Nos remerciements vont à Olga Anokhina, à Daniel Ferrer ainsi qu’à Emilio Sciarrino sans qui cet article ne serait pas parvenu à bonne fin. $(* *)$ «... même s'il est habituel de ne pas considérer le noir, le blanc comme étant des couleurs, l'un est en effet ténèbres et l'autre lumière, l'un ôte et l'autre engendre, moi je ne veux pas les laisser de côté, car en peinture ils sont essentiels, on sait que la peinture est formée d'ombre et de lumière, de clair et d'obscur. » Pour le texte original cité en exergue, cf. Trattato della pittura: tratto da un codice della Biblioteca Vaticana, vol. I, Leonardo da Vinci, Guglielmo Manzi, Éditeur Stamperia de Romanis, 1817, p. 125-126. Original provenant de la bibliothèque de l'État de Bavière. Numérisé le 17 février 2010. En ligne : <https://books.google.fr/books?id=-w1BAAAAcAAJ\&hl =fr\&source=gbs_navlinks_s> (consulté en décembre 2017).

1. Dans le métatexte Temas y estructuras [Thèmes et structures], Lekuona se présente ainsi : «Mon métier est d'être professeur de littérature et traducteur vers le basque, à intervalles réguliers j'ai fait des incursions dans le champ de la poésie érudite [...] » (Ibilaldia - Itinerario (1950-1990) [Itinéraire], Servicio Editorial/Argitalpen Zerbitzua, Universidad del País Vasco - Euskal Herriko Unibertsitatea, Leioa, 1996, p. 46).

2. On peut mentionner à ce titre la naissance de l'université du Pays basque ou Universidad del País Vasco-Euskal Herriko Unibertsitatea (1980) localisée sur trois campus (Vitoria, Bilbao, Saint-Sébastien).

3. Julio César Santoyo, «Autotraducciones: Una perspectiva histórica», Meta, vol. L, n 3, août 2005, p. 864. Sauf indication contraire, ici et plus loin, c'est nous qui traduisons en français.

4. Ibid., p. 866.

5. Rainier Grutman, «L'autotraduction : de la galerie de portraits à la galaxie des langues », Glottopol, $\mathrm{n}^{\circ}$ 25, 2015, p. 14-30. en ligne : < http://glottopol. univ-rouen.fr/telecharger/numero_25/gpl25_01grutman.pdf > (consulté en décembre 2017).

6. Christian Lagarde, «De l'individu au global : les enjeux psycho-sociolinguistiques de l'autotraduction littéraire», Glottopol, op. cit., p. $31-46$. 
Zaitouni-Chapin 7 pour l'aire ibérique, ainsi que les études d'Arantza Manterola-Arrizabalaga ${ }^{8}$, de Cristina JarillotIbon Uribarri ${ }^{9}$, de Miren Ibarluzea-Marijose Olaziregi 10 et d'Aiora Jaka11, consacrées au champ littéraire contemporain en Pays basque péninsulaire, montrent que ce phénomène s'est beaucoup amplifié ces quinze dernières années.

Les écrivains divergent sur le fait de s'autotraduire en basque ${ }^{12}$. Ceux qui inscrivent l'autotraduction dans des rapports de force opposant un dominant à un dominé ont une attitude de défiance vis-à-vis de l'autotraduction en castillan, voire optent pour la stratégie du «court-circuitage » comme l'exprime l'écrivain Iban Zaldua :

Même si chez nous la situation officielle de la langue basque s'est améliorée quelque peu, au moment d'être traduit, c'est le «court-circuitage» de l'espagnol qui continue à avoir le plus de succès chez les bascophiles, surtout chez ceux qui professent un nationalisme quelque peu accentué. En fin de compte, ne pas passer par l'espagnol est une manière de se détacher du lien «colonial», une expression d'autonomie culturelle et politique ${ }^{13}$.

Que «l'autotraduction devienne systématique n'est pas une bonne chose pour la littérature basque», déclare par ailleurs l'écrivain et critique Ur Apalategi, car «il s'agit d'une question d'ordre politique et éthique ${ }^{14} »$. Joseba Sarrionaindia, auteur basque vivant à Cuba, compare avec causticité les écrivains d'expression basque qui s'autotraduisent en castillan aux «chèvres de Leila» du détroit de Gibraltar qui vivaient dans une indifférence générale (et une paix royale) jusqu'à ce que par malheur on s'intéressât un jour à elles 15 .

D'autres écrivains comme Harkaitz Cano ou Kirmen Uribe, qui établissent «une séparation claire entre création et traduction littéraire» et qui préfèrent se faire traduire en castillan par des traducteurs professionnels pour «se consacrer uniquement au travail créatif $16_{»}$, rejoignent la position de Miguel del Toro, pour qui s'autotraduire est «une perte de temps 17 ». Ces mêmes auteurs ont pu cependant concevoir, à d'autres moments, l'autotraduction comme un acte de création permettant un retour sur le texte originel. Ainsi, Kirmen Uribe a autotraduit ses premiers textes poétiques. Harkaitz Cano, s'il devait s'autotraduire, écrirait davantage une «version qu'une traduction», en étant tenté «de réécrire, de réécrire à l'infini 18 ». Mariasun Landa, auteure d'expression basque spécialisée dans la littérature pour la jeunesse, pense que l'autotraduction est comme «quitter une maison pour en habiter une autre [...] un acte d'hospitalité, [...] un passage sur la voie de l'habitabilité

7. Nayrouz Zaitouni-Chapin, Le Bilinguisme en littérature : l'autotraduction espagnol-français à la lumière du cas d'Agustín Gómez Arcos, thèse de doctorat, université Michel de Montaigne - Bordeaux III, 2015. En ligne : < https://tel.archives-ouvertes.fr/tel-01258053> (consulté en décembre 2017)

8. Elizabete Manterola Agirrezabalaga, Euskal Literatura beste hizkuntza batzuetara itzulia, Bernardo Atxagaren lanen itzulpen moten arteko alderaketa [La Littérature basque traduite à certaines langues. Variantes entre diverses sortes de traduction dans les travaux d'Atxaga], thèse de doctorat, Universidad del País Vasco/Euskal Herriko Unibertsitatea, 2014. 9. Cristina Jarillot-Ibon Uribarri, «Politiques de la traduction dans un environnement multilingue : le cas basque», Traduction et partages : que pensons-nous devoir transmettre, Actes du XXXVIIe Congrès de la SFLGC les 27-29 octobre 2011, 2014. En ligne : < http://www.voxpoetica.com/sflgc/actes/traduction/4.5.\%20Jarillot\%20Uribarri.pdf> (consulté en décembre 2017).

10. Miren Ibarluzea et Mari Jose Olaziregi, «Autonomización y funciones del subcampo de la traducción literaria vasca contemporánea: una aproximación sociológica», Pasavento. Revista de Estudios Hispánicos, vol. IV, $\mathrm{n}^{\circ} 2$, 2016, p. 293-313 (<http://www.pasavento.com/pdf/02Ibarluzea_ Olaziregi.pdf $>$ [consulté en décembre 2017]).

11. Aiora Jaka, «Contribution de l'étude de la traduction des contextes minorisés à la méthodologie de la recherche en traductologie : le cas basque », Érudit, Traduction, Terminologie, Rédaction TTR 242, numéro thématique Cartographie des méthodologies en traduction, vol. XXIV, $\mathrm{n}^{\circ} 2$, 2011, p. 120. En ligne : < https://www.erudit.org/fr/revues/ttr/2011v24-n2-ttr0392/1013397ar.pdf> (consulté en décembre 2017).

12. Karlos Cid Abasolo, «Autotraducción literaria desde el vasco», Revista de Filología Románica, Anejo IX, 2015. En ligne : < https:// revistas.ucm.es/index.php/RFRM/article/viewFile/48187/45072 > (consulté en décembre 2017).

13. Les propos d'Iban Zaldua sont cités par Karlos Abasolo, op. cit., p. 179.

14. «I think it was not a good thing for Basque literature that self-translation became systematic: it is a political and ethical question». Miren Ibarluzea, «The Translation Habitus of Contemporany Basque Writers, Estudios de Traducción», vol. V, 2015, p. 66. En ligne : < http://revistas. ucm.es/index.php/ESTR/article/view/49410> (consulté en décembre 2017).

15. Les propos de Sarrionaindia sont cités par Karlos Abasolo, op. cit., p. 179.

16. Miren Ibarluzea et Mari Jose Olaziregi, op. cit., p. 300. Cf. également Miren Ibarluzea, «The Translation Habitus of Contemporary Basque Writers », op. cit., p. 66.

17. Cité par Christian Lagarde «De l'individu au global : les enjeux psycho-sociolinguistiques de l'autotraduction littéraire», op. cit., p. 31. 18. Karlos Abasolo, op. cit., p. 183. 
du monde 19 ». Selon Bernardo Atxaga, écrivain basque mondialement connu et traduit dans vingt-trois langues, à qui nous avons posé la question dans le cadre de cet article, il s'agit d'une question «complexe» non seulement pour des raisons linguistiques, mais parce qu'interfèrent également des problématiques historico-sociales concernant le statut dominé de cette même langue : l'autotraduction s'avère alors la «meilleure des options quand un auteur connait les deux langues en lice»; c'est ce que pensent aussi Iban Zaldua 20 et Unai Elorriaga, pour qui l'autotraduction est la seule voie qui permette d'obtenir une bonne version 21 .

Juan Mari Lekuona a toujours accordé une place importante à la traduction dans sa pratique poétique sans toutefois jamais mettre en lumière sa pratique autotraductive. Chez lui, le mot «traduire» recouvre à la fois l'activité de «traduire en basque de la poésie écrite en d'autres langues» mais aussi de «traduire [s]a propre poésie en [s]on autre langue, le castillan ». C'est ce que laissent entendre en sourdine les propos suivants tirés d'une interview réalisée par son ami et ancien élève, l'écrivain Joxe Auxtin Arrieta :

Dur d'être un bon poète basque dans nos contrées si on est incapable de traduire en basque de la poésie écrite en d'autres langues. Le fait de traduire la poésie étrangère en langue basque est d'un secours considérable (pour l'écrivain). Il doit en être de même en prose. Si la relation devient intime, aucun risque de colonialisme. Car la culture ne se limite pas à ce que vous ou moi réalisons, elle ne nous appartient ni à vous ni à moi, il s'agit d'un patrimoine universel. Et c'est là que la traduction remplit pleinement sa fonction 22 .

La trajectoire poétique de Juan Mari Lekuona a été ponctuée par quatre ouvrages en langue basque : Mindura gaur [Souffrance d'aujourd'hui], recueil ronéotypé, paru en 1966, dont les matériaux serviront à l'échafaudage d'un second recueil publié en 1973 Muga beroak [Limites ardentes]; Ilargiaren eskolan [À l'école de la lune] (1979) et, enfin, Mimodramak eta Ikonoak [Mimodrames et Icônes] (1990)23.

Lekuona a été longtemps peu disert sur sa pratique d'écriture mais les métatextes auctoriaux ${ }^{24}$ permettent au lecteur d'en apprendre davantage sur sa démarche poétique. En revanche, sa pratique autotraductive en espagnol, employée lors de sa création de Mimodrames et Icônes en langue basque, demeure ignorée de tous (lecteurs, critiques, traducteurs allogènes, médias) car l'auteur ne se référa jamais au plurilinguisme de son écriture. Or, les brouillons de Mimodrames et Icônes montrent que l'œuvre dotée de ses avant-textes n'est pas une forme «close», «autosuffisante», «monolingue», comme la version publiée le laissait entendre : sa lecture acquiert ainsi une nouvelle perspective.

\section{L'espagnol, langue-miroir dans l'œuvre basque Mimodrames et Icônes}

Nous disposons d'un dossier comportant plusieurs états génétiques en langue basque dont certains ont leur équivalent (souvent partiel) en espagnol. Nous nous interrogerons donc sur la nature et sur la portée de l'écriture en langue espagnole au sein d'une œuvre qui ne sera publiée initialement qu'en langue basque.

Ainsi qu'il nous le confia plusieurs fois lors d'entretiens à bâtons rompus sur les enjeux de la création poétique contemporaine en langue basque, Lekuona conseillait la pratique de l'autotraduction (qu'il désignait comme étant l'acte de «(se) traduire» [itzultze]) afin d' «améliorer la qualité esthétique» du texte original, étant entendu de manière tacite qu'un auteur écrivant en langue minorée

19. Mariasun Landa, «La autotraducción como reescritura creativa», dans S. Hibbs et M. Martinez (dir.), Traduction, adaptation, réécriture dans le monde hispanique contemporain, Toulouse, Presses universitaires du Mirail, 2006, p. 52-60 cité par Christian Lagarde (op. cit., 2015, p. 42) qui a traduit du castillan au français le passage rapporté ici.

20. Karlos Cid Abasola, op. cit., p. 180.

21. Miren Ibarluzea, op. cit., p. 66.

22. Cf. Juan Mari Lekuona hurbiletik (1927-2005) [Du côté de chez Juan Mari Lekuona (1927-2005)], Oihartzungo udala [Mairie de Oiartzun], Euskaltzaindia [Académie de la langue basque], Oiartzun, 2008, p. 175. 23. Lekuona préparait un cinquième opus poétique resté inachevé. Cf. Juan Mari Lekuona, Ibaiak basamortuan [Fleuves dans le désert], Édition et introduction par Jon et Paulo Kortazar, Universidad del País Vasco - Euskal Herriko Unibertsitatea, Bilbao, 2008.

24. Trois métatextes, regroupés sous le titre général de Neure poetikari buruz [Vis-à-vis de ma poétique], forment le paratexte préfaciel de l'ouvrage anthologique bilingue (basque/espagnol) intitulé Ibilaldia Itinerario (1950-1990) [Itinéraire] (1996) (voir supra). Par ordre d'apparition dans la préface, il s'agit de Poetika baten bilakabidea eta azpiko hariak [Devenir d'une poétique et trame secrète] (1996), rédigé en basque; Temas y estructuras [Thèmes et structures] (1986; 1996), rédigé en espagnol; Acotaciones [Annotations] (1994; 1996), rédigé en espagnol. Cf. op. cit., p. 10-57. 
ou «régionale» est nécessairement bilingue : dans le cas du basque, respectivement basque-espagnol ou basque-français.

$\grave{A}$ aucun moment il ne s'agissait pour lui de pratiquer l'autotraduction dans l'objectif de publier deux versions du même texte, comme c'est pourtant le cas de la plupart des écrivains qui s'autotraduisent ${ }^{25}$. L'horizon d'attente de la contrainte était uniquement esthétique. Lekuona disait que la traduction permettait de «mieux cibler» les faiblesses du texte en cours d'élaboration. D'ailleurs, il n'était pas question d'opposer une «langue cible» versus une «langue source», mais bien plutôt de penser en termes de «réflexion» au sens premier : «renvoyer par réflexion dans la direction d'origine ou dans une autre direction».

L'utilisation de l'espagnol couvre les deux sens du mot réflexion pour Lekuona. L'espagnol est ainsi à la fois «surface réfléchissante» et outil dynamique qui renvoie «par réflexion dans la direction d'origine», vers le basque, afin d'améliorer le texte d'origine et de continuer le processus créatif. Cette pratique scripturale lors du processus de création fait penser à l'utilisation du miroir en peinture comme facteur de distanciation.

La deuxième langue, utilisée comme médium permettant de s'éloigner et ainsi de mieux «voir», est donc bien plus qu'une simple «traduction» par l'auteur de son propre texte, elle devient «miroir». La langue poétique de Lekuona, au niveau du lexique, comporte d'ailleurs de nombreux éléments traductifs. Les emprunts, par exemple, sont nombreux, à commencer par le titre Mimodramak eta Ikonoak [Mimodrames et Icônes], compréhensible pour toute personne connaissant l'espagnol bien entendu, mais plus généralement les langues romanes (alors qu'il est d'usage de souligner la différence typologique du basque en mettant en avant son statut d' «isolat linguistique » au sein des langues indo-européennes).

Il est important de préciser que les autotraductions en espagnol figurent uniquement dans la phase pré-rédactionnelle et rédactionnelle de Mimodrames et Icônes. À titre d'exemple, la première chemise du Dossier 1 intitulée «Lehen hurbilketak (1982-1987)» [Premières approches (1982-1987)], comprend des notes de régie, des plans programmatiques manuscrits ainsi que cinq poèmes dont deux manuscrits exclusivement en langue basque et trois autres poèmes dactylographiés et autotraduits fragment

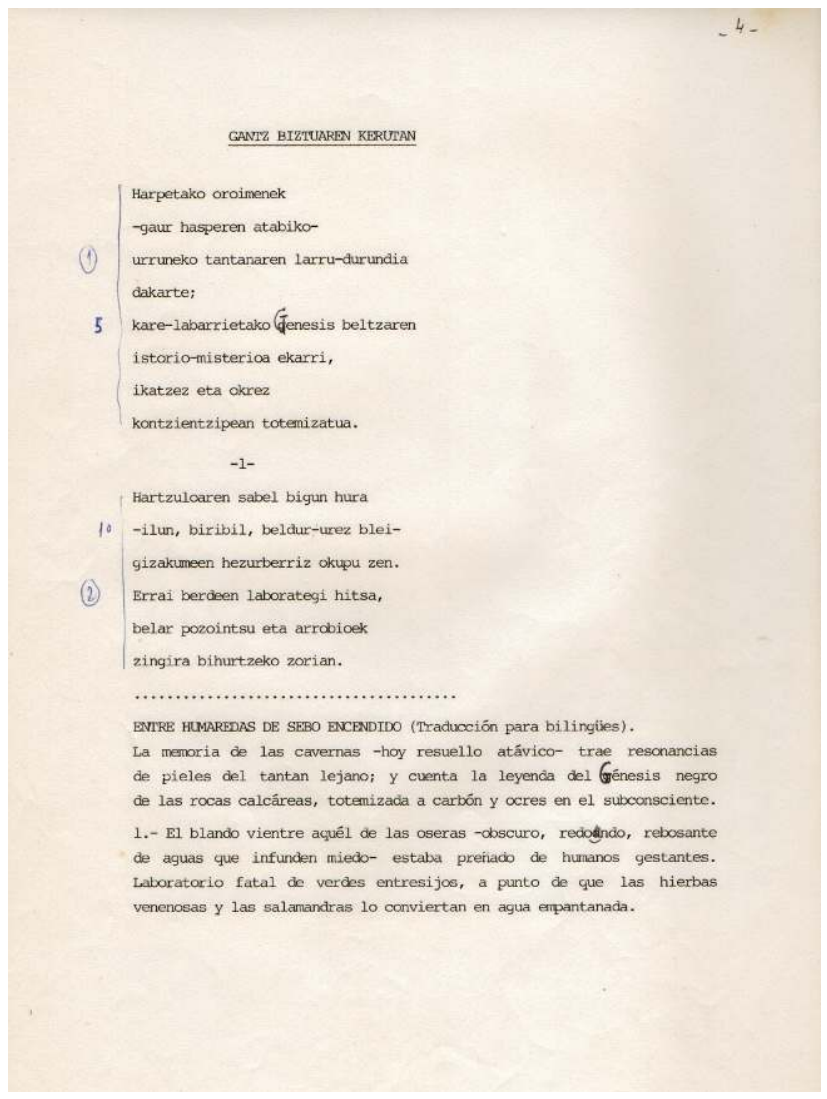

Fig. 1 : Le poème Gantz biztuaren kerutan - Entre humaredas de sebo encendido [Dans les odeurs âcres de graisse ardente]. Dossier 1 «Lehen hurbilketak (1982-1987)» [Premières approches (1982-1987)], archive personnelle d'Aurelia Arcocha-Scarcia

par fragment, au fur et à mesure de l'avancement de l'écriture (fig. 1).

Au fur et à mesure de l'avancement du processus créatif, la version espagnole disparaît, ce qui semble indiquer que, n'étant pas considérée comme importante pour la publication, elle ne constituait qu'une étape provisoire de la genèse.

Une autre particularité doit être soulignée : la traduction des vers basques en espagnol se fait en prose, ce qui accentue encore une dissymétrie dans l'utilisation des langues et un rôle instrumental de la traduction espagnole. La traduction en prose permettrait en effet de saisir le «contenu» du texte en basque sans versification.

25. La question des traductions allogènes de Mimodrames et Icônes en espagnol, certaines étant des traductions collaboratives, ne sera pas abordée ici. Se reporter à l'ouvrage Ibilaldia - Itinerario (1950-1990) [Itinéraire (1950-1990)], op. cit., p. 195-290, pour lire les poèmes de Mimodrames et Icônes traduits en espagnol, ainsi qu'à la page de titre de ce même ouvrage où figurent les noms des différents traducteurs. 

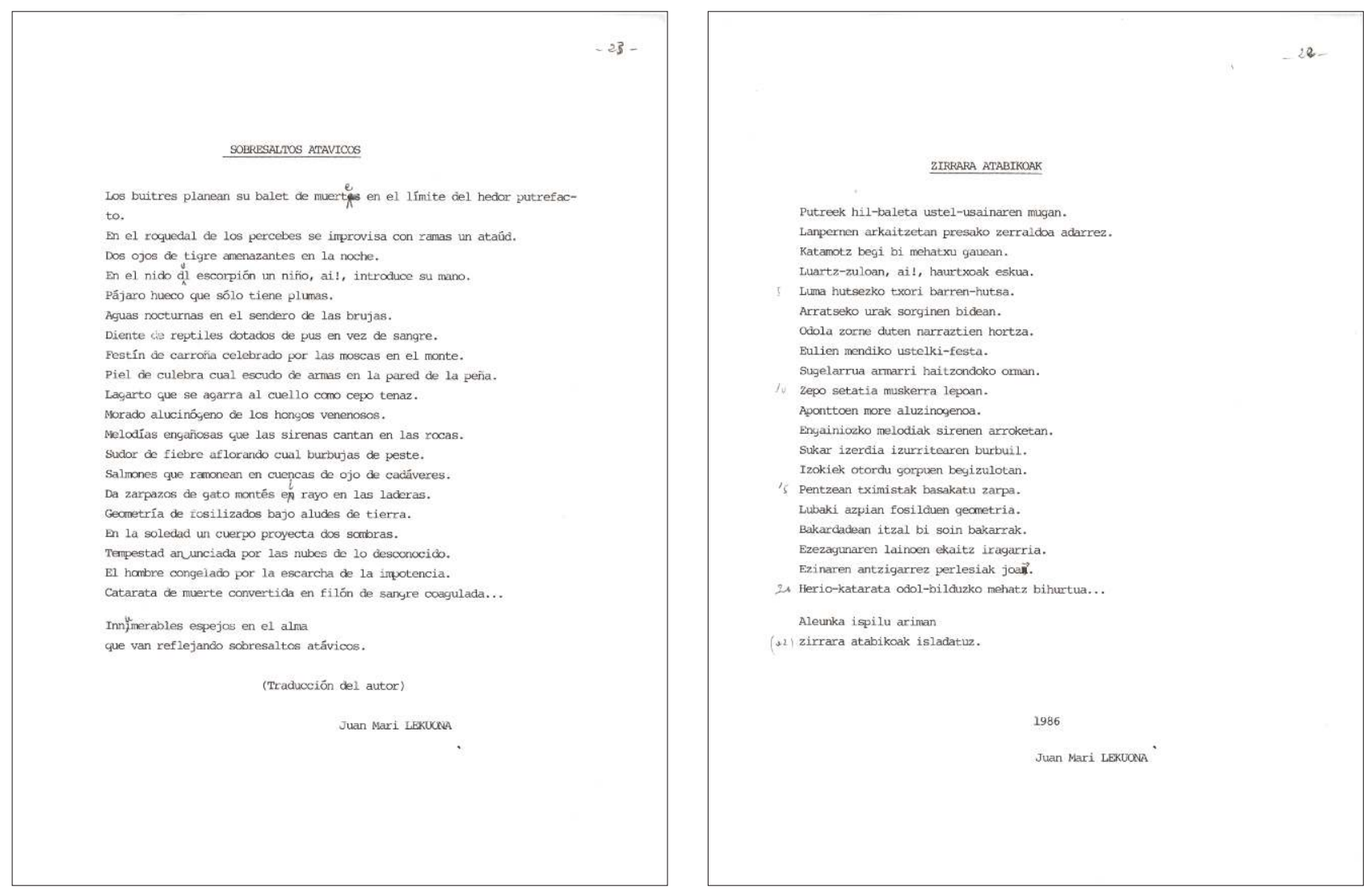

Fig. 2 : Poème de 1986, Zirrara atabikoak-Sobresaltos atávicos [Sursauts ataviques], dactylographié dans deux mises en page équivalentes sur des feuilles volantes (rectos).

Dossier 2 intitulé «Lehen zirriborroa... » [Premiers brouillons], «Uztaren azkenetan bukatua» [Terminé fin 1989]

Cela n'empêche pas Lekuona de mettre en pratique, sensiblement au même moment, un autre système, en diptyque : le poème en basque et le poème autotraduit en espagnol apparaissent dactylographiés sur les rectos de deux feuilles volantes, dans deux mises en page équivalentes (fig. 2).

La machine à écrire aide Lekuona à prendre de la distance, à «dépersonnaliser ${ }^{26}$ » son texte. Lekuona applique ce «jeu de miroirs» dès les premières étapes de la phase rédactionnelle (Dossier 1, voir supra).

Dans Obertura-Obertura [Ouverture], la mise à distance introduite par la transcription dactylographique réactive le processus de création en basque qui se poursuit à la main (fig. 3 recto), lequel stimule à son tour l'application d'une deuxième strate réfléchissante, celle de l'autotraduction manuscrite en espagnol en amplifiant ainsi le mouvement autotraductif circulaire 27 (fig. 3 verso).

Lors de l'étape plus tardive d'élaboration des poèmes de Mimodrames et Icônes avec autotraductions (Dossier 3 daté des années 1989-1990), Lekuona élabore des versions face à face, en basque et en espagnol, grâce à un grand cahier à anneaux qui permet une interaction des deux langues en miroir (voir infra).

\section{Spatialité et temporalité}

Quand le texte en basque et le texte autotraduit se retrouvent sur la même page manuscrite, ils n'occupent pas hiérarchiquement les mêmes espaces. La primauté est toujours donnée au texte en basque. Ainsi, dans les textes

26. $C f$. Catherine Viollet, «Écriture mécanique, espaces de frappe. Quelques préalables à une sémiotique du dactylogramme», Genesis, numéro thématique Sémiotique, $\mathrm{n}^{\circ} 10,1996, \mathrm{p} .208$. En ligne : < http:// www.persee.fr/doc/item_1167-5101_1996_num_10_1_1075> (consulté en décembre 2017).

27. Olga Anokhina, «Cas limites d'autotraduction : cercle, spirale, chaos », dans Esa Hartmann et Patrick Hersant (dir.), Au miroir de la traduction : avant-texte, intratexte, paratexte, Paris, Éditions des archives contemporaines, coll. «Multilinguisme, traduction, création», 2018 (sous presse). 

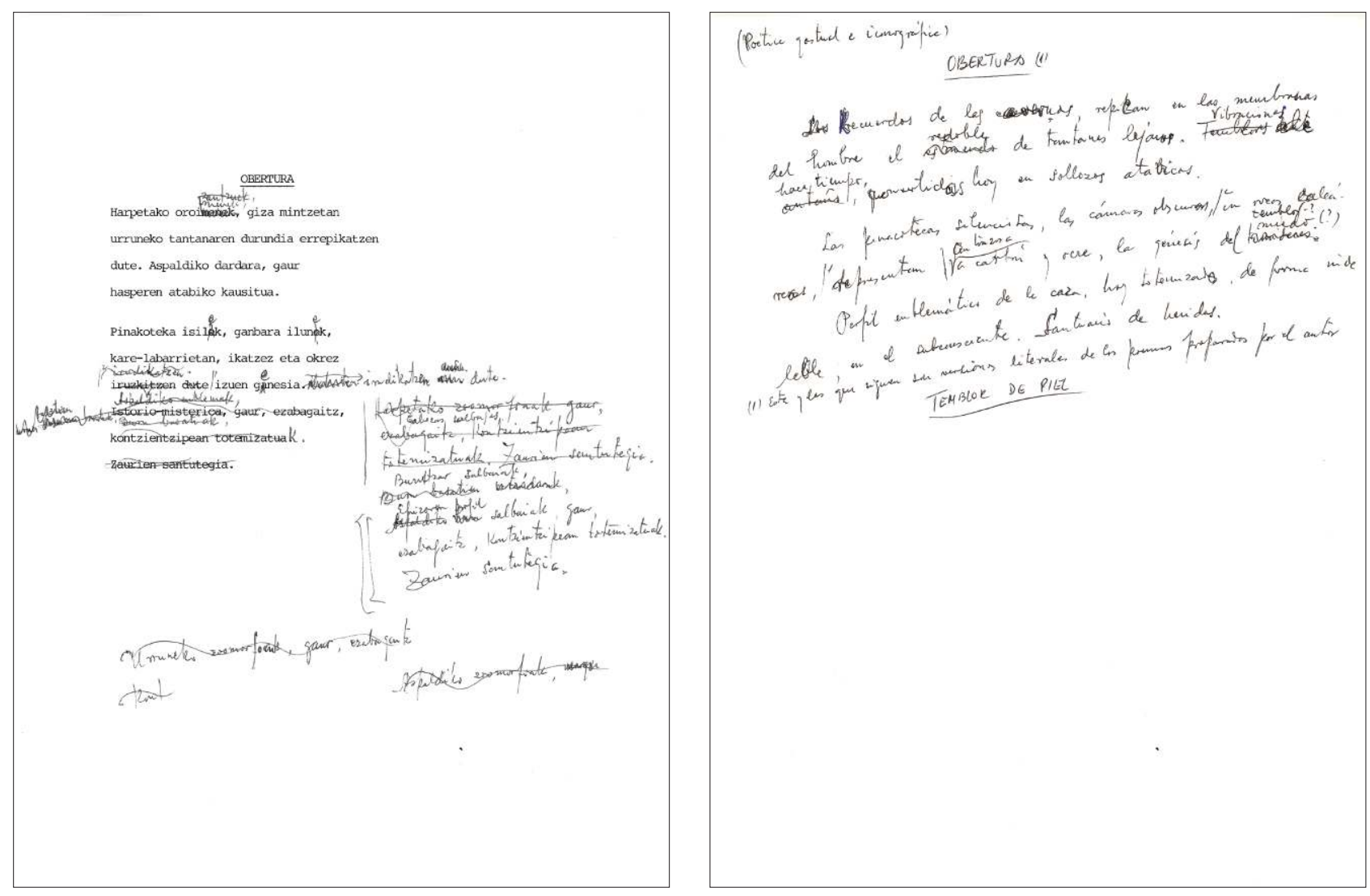

Fig. 3 : Obertura-Obertura [Ouverture]. Dossier 2.

Dactylogramme en basque avec la campagne de correction manuscrite (recto) et l'autotraduction manuscrite (verso)

autotraduits et dactylographiés de 1986-1987, le texte-source en basque occupe l'espace central de la page, l'autotraduction en espagnol s'insérant dans la marge droite. Vu la disposition spatiale des deux textes, il semble évident que le texte basque était le premier à occuper l'espace, le texte autotraduit n'intervenant qu'une fois celui-ci couché sur le papier.

L'observation de plusieurs états génétiques du dossier montre bien que Lekuona commence toujours par travailler d'abord le texte en basque et que ce n'est qu'à partir d'un certain stade qu'il décide de faire une version en espagnol.

Certains détails techniques, comme la datation, confirment le caractère consécutif du processus créatif plurilingue chez Lekuona. Par exemple, dans le poème Oihanean [Dans la forêt], une première campagne d'écriture date du 4 septembre 1989 où le texte en basque s'élabore à droite, de haut en bas au fur et à mesure des reprises (comme l'indiquent les flèches directionnelles). Il est ensuite autodratuit en espagnol à gauche ce même jour. Ces deux versions sont ensuite barrées. Sur les deux feuilles suivantes du cahier mis à plat, Lekuona reprend la version en basque qu'il met au propre et retravaille sur la partie droite le 4 septembre, puis il l'autotraduit à nouveau en espagnol le lendemain 5 septembre sur la partie gauche du cahier. Il y a donc un jour de décalage entre les deux versions (fig. 4a et 4b).

Ce texte sera ensuite repris ultérieurement à la main, exclusivement en basque, sans autotraduction, sur l'une des feuilles volantes insérées à la fin du cahier rouge à spirales (fig. 5 recto et verso).

Le caractère consécutif se voit aussi très nettement dans le cas des campagnes d'écriture plus tardives d'OberturaObertura [Ouverture] (Dossier 3). Tout commence par une première version en langue basque sur la page de droite qui est retravaillée de haut en bas (flèches directionnelles) (fig. 6a), sans aucune autotraduction sur la page de gauche. Puis vient une seconde campagne datée du 30 août (fig. 6b), où le texte en basque (page de droite) est autotraduit frontalement sur la page de gauche, puis les deux versions sont barrées. L'autotraduction redynamise le poème en basque qui est repris sur la partie droite sous la version précédente puis barré à son tour (fig. 6b, détails). 


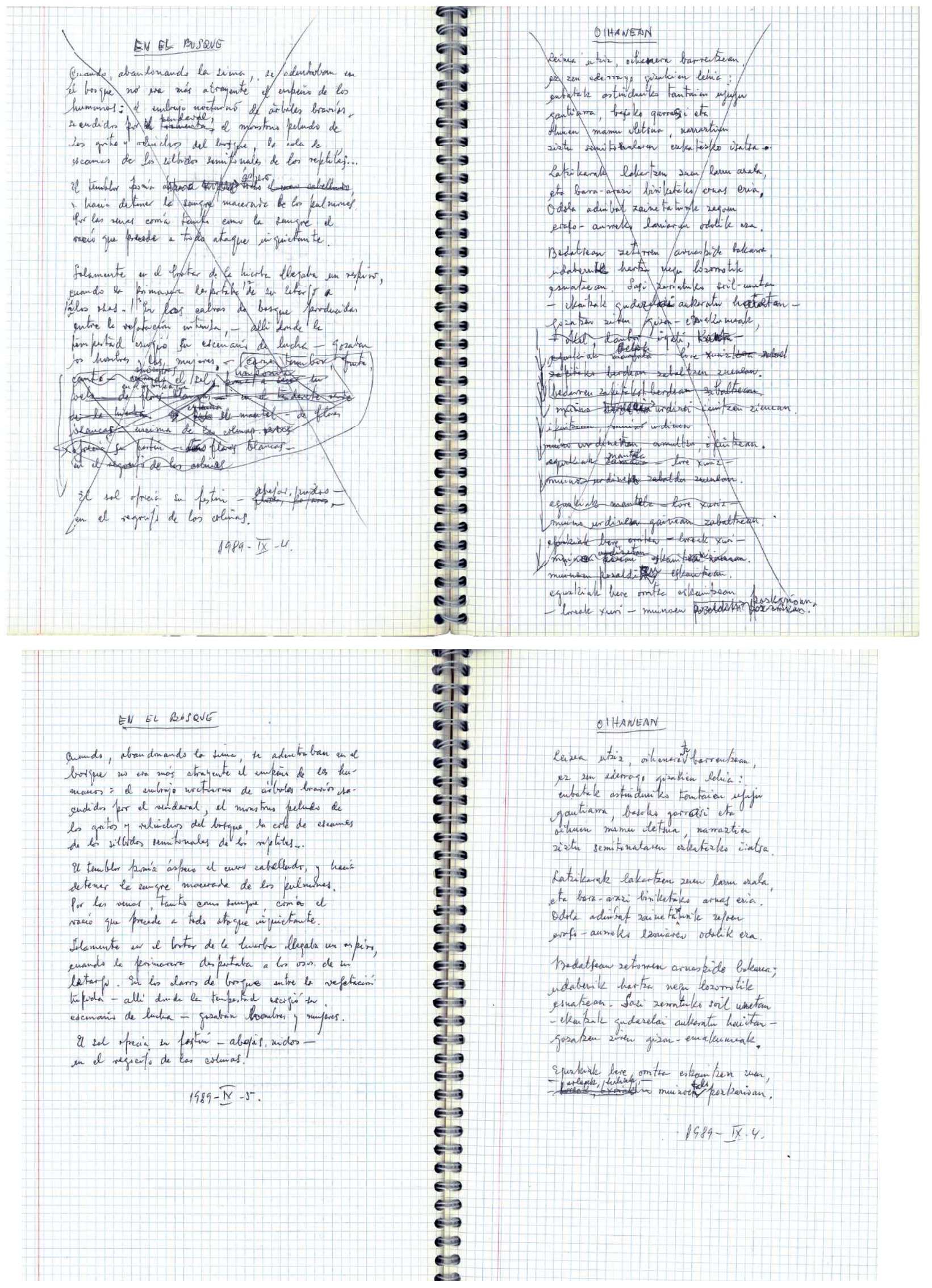

Fig. 4a et 4b : Poème Oihanean-En el bosque [Dans la forêt]. Dossier 3 intitulé «Bigarren zirriborroa (1989ko Abuztutik 1990ko otsaila arte») Deuxième brouillon (depuis août 1989 jusqu'en février 1990).

À droite partie en langue basque datée du 4 septembre 1989, à gauche partie en espagnol datée du 5 septembre 1989 


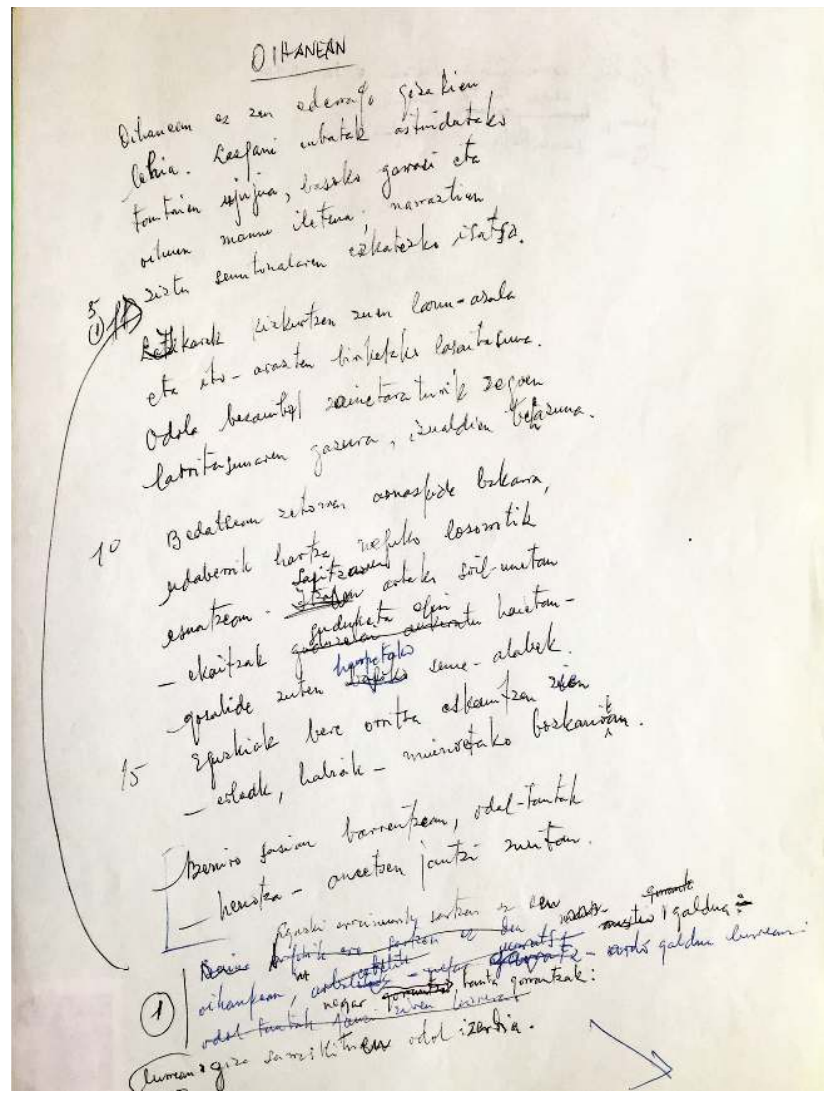

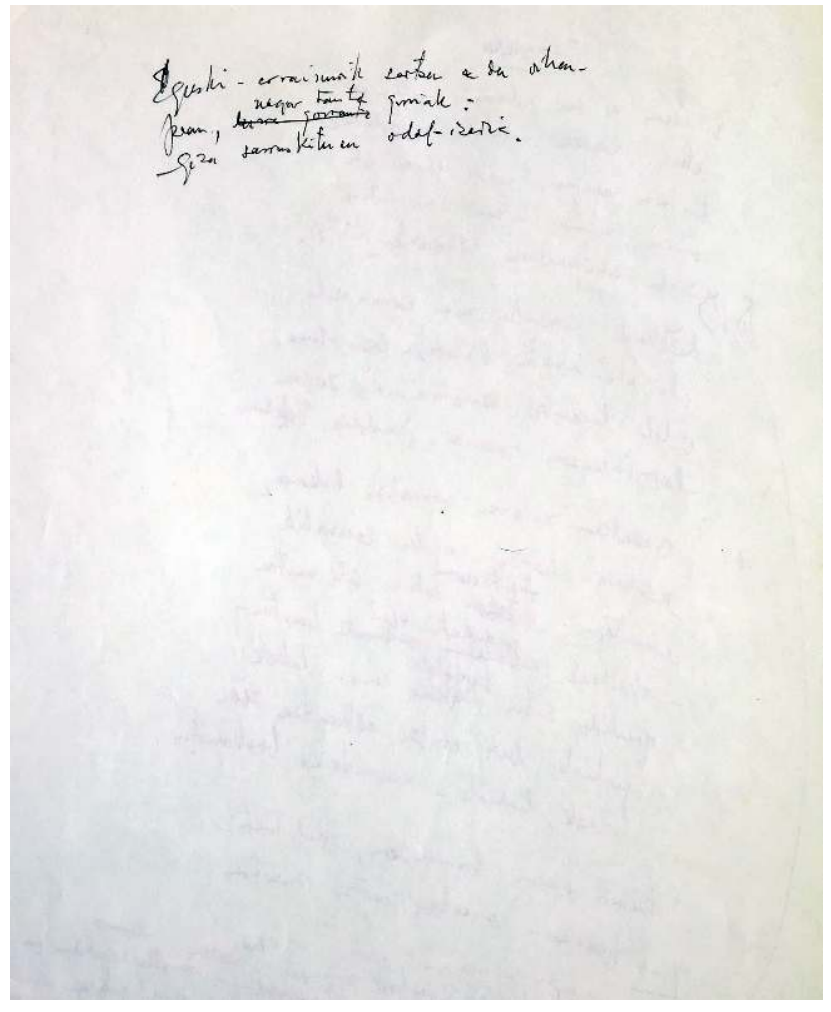

Fig. 5 : Recto à gauche et verso à droite :

Poème Oihanean-En el bosque [Dans la forêt]. Dossier 3
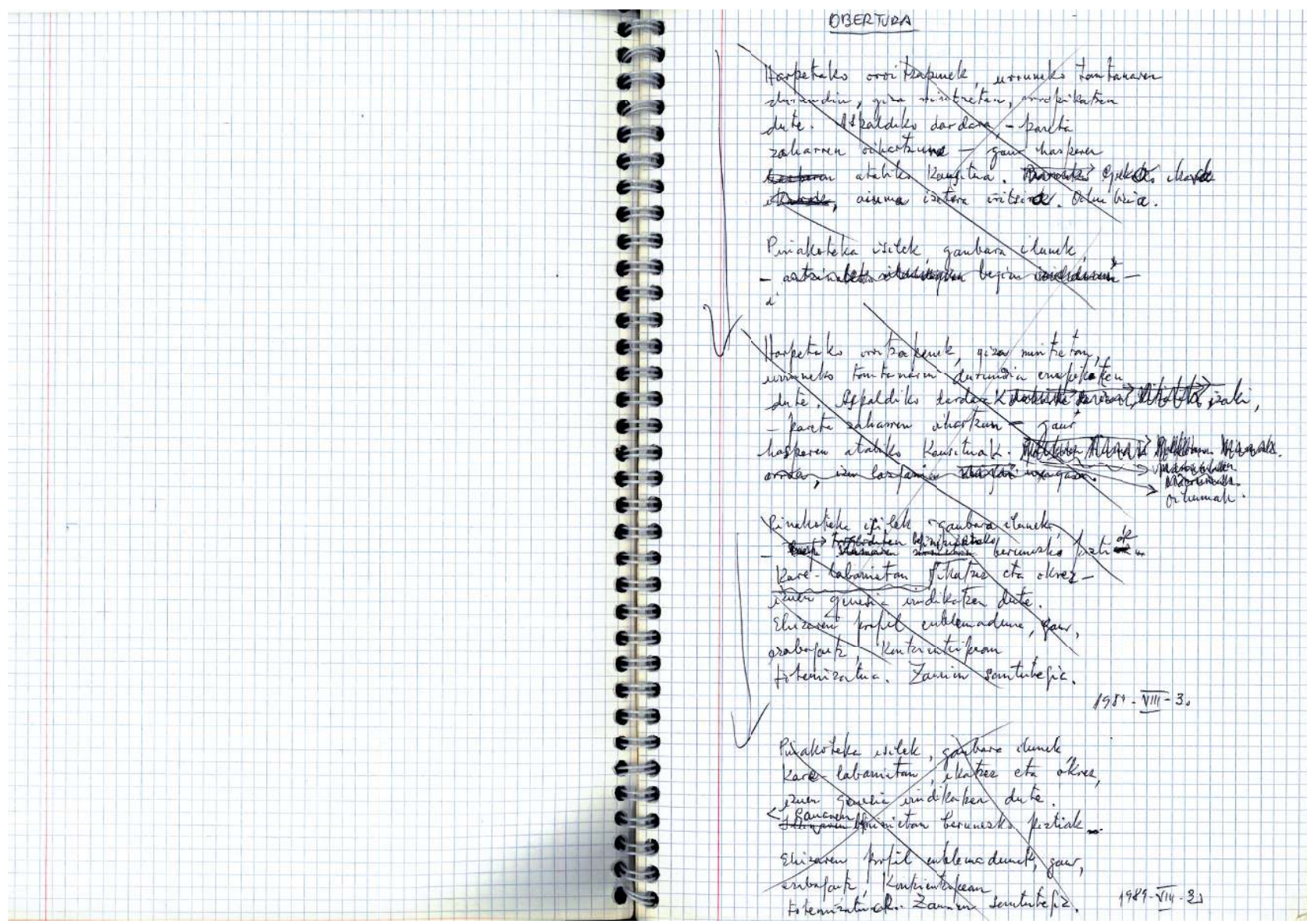

Fig. 6a : Première campagne d'écriture du poème Obertura [Ouverture]. Dossier 3 
Fig. $6 \mathrm{~b}$ : Deuxième campagne d'écriture du poème Obertura [Ouverture]. Dossier 3
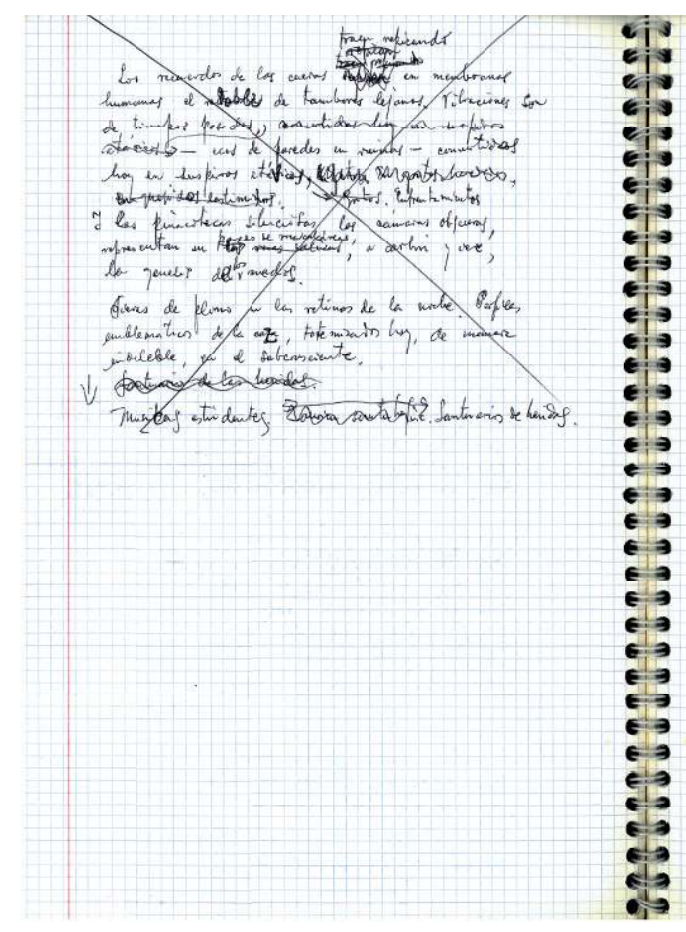

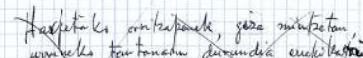

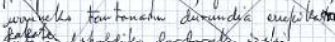

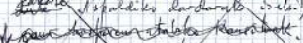

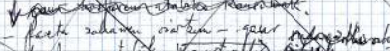

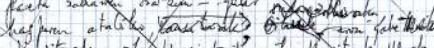

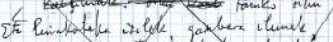
kave-laterictam; sthation cto dipe

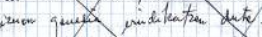

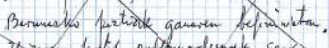

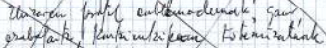
zamin sontulefir DPERT TOS

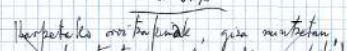

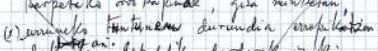

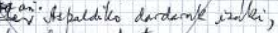

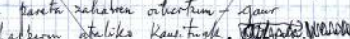
oledertar dinlesiale.

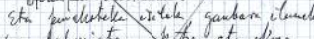

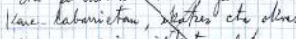

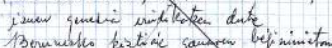

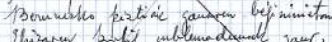

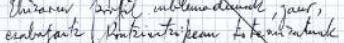

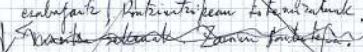

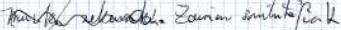

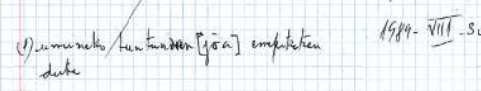

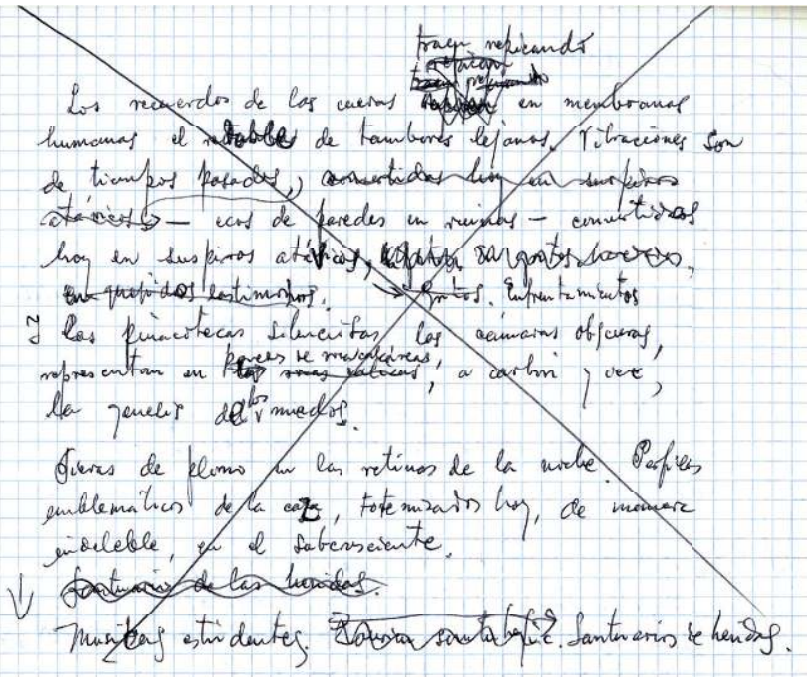

Fig. $6 \mathrm{~b}$ : Deuxième campagne d'écriture du poème Obertura [Ouverture] (détails). Dossier 3

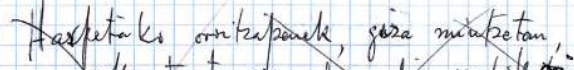

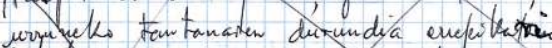

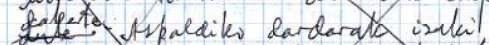

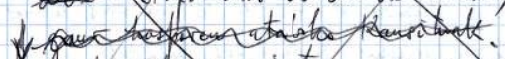

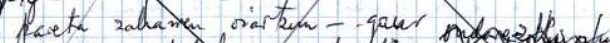

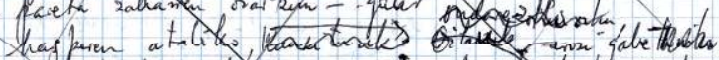

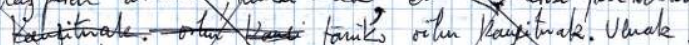

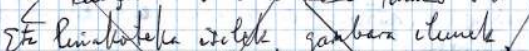

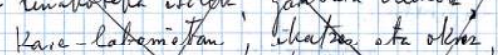

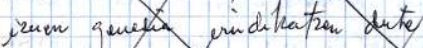

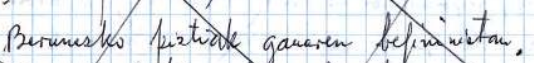

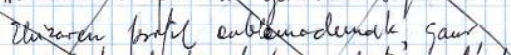

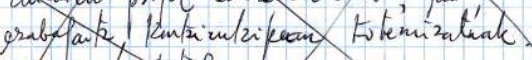
zalonim soutatefir.

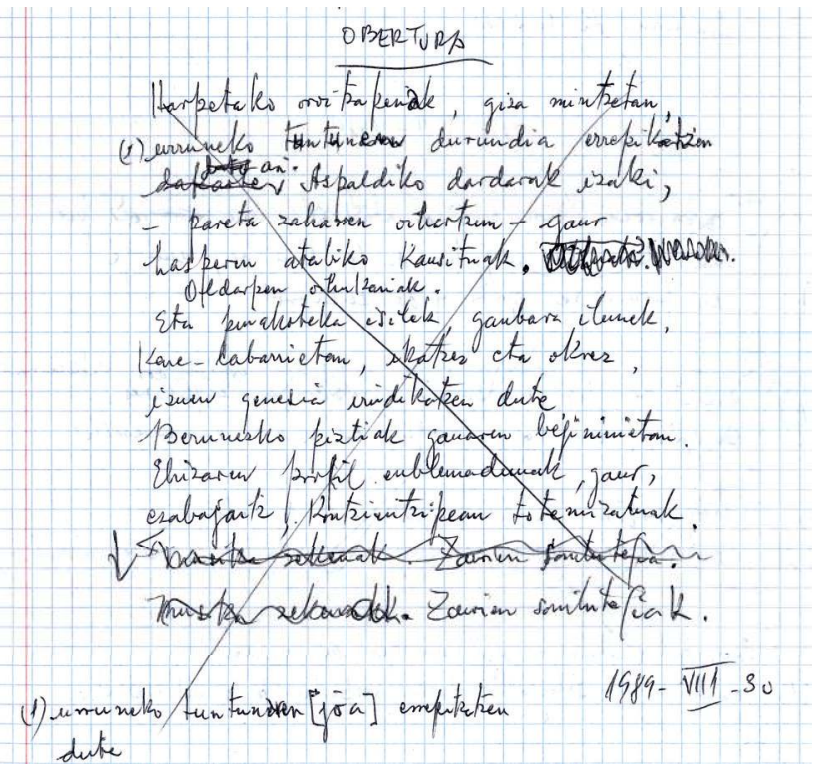



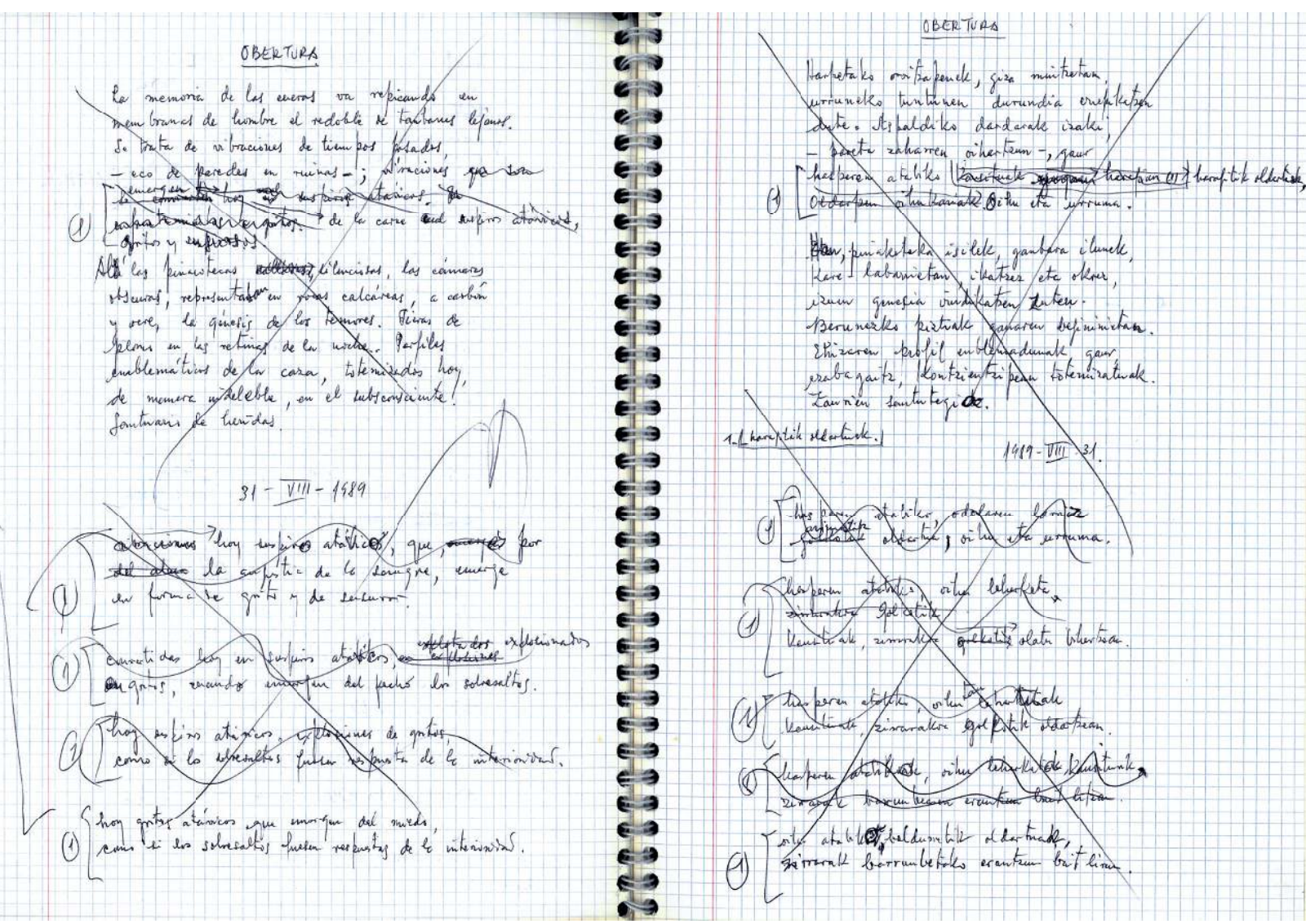

Fig. 6c : Troisième campagne d'écriture du poème Obertura [Ouverture]. Dossier 3

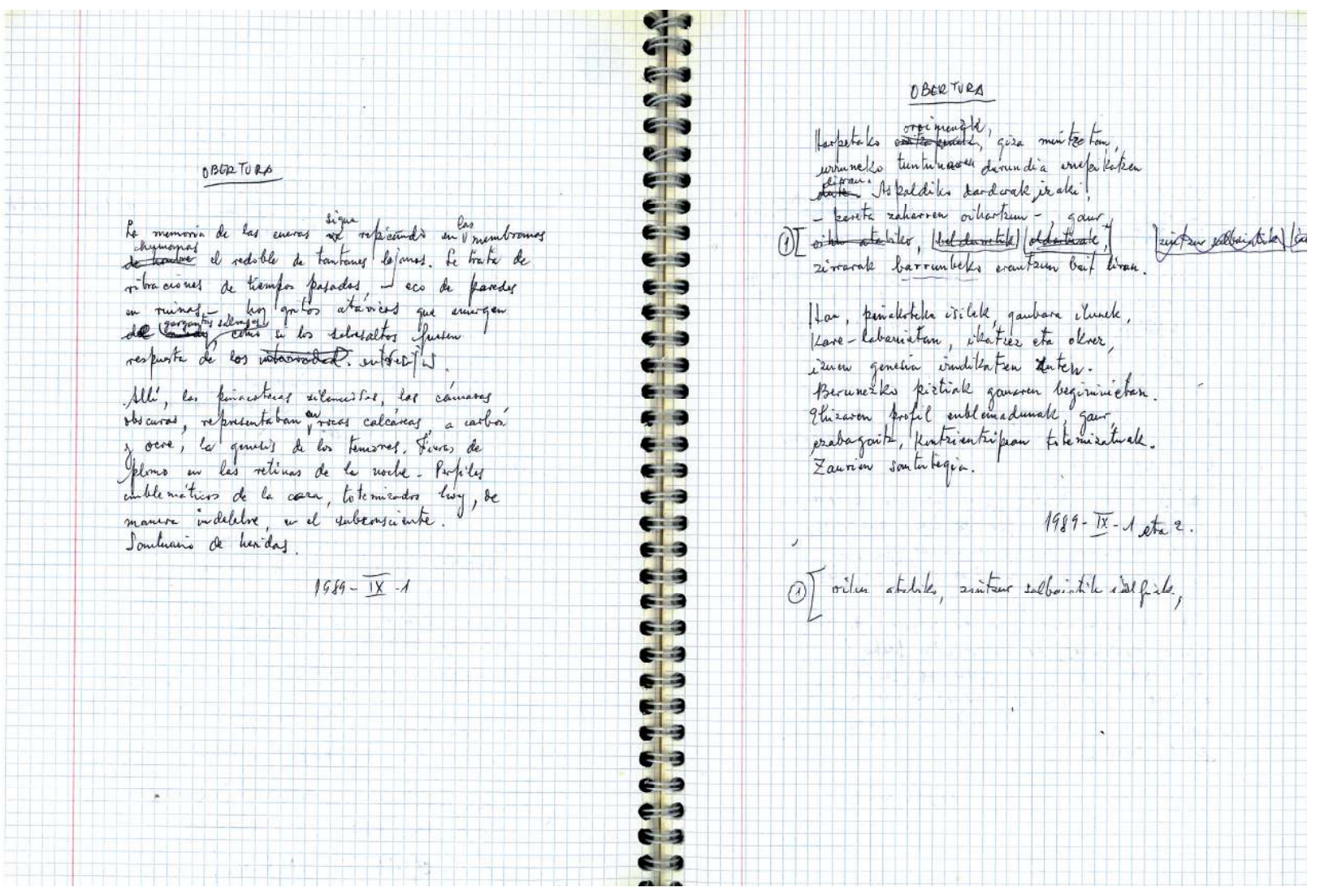

Fig. 6d : Quatrième campagne d'écriture du poème Obertura-Obertura [Ouverture]). Dossier 3 


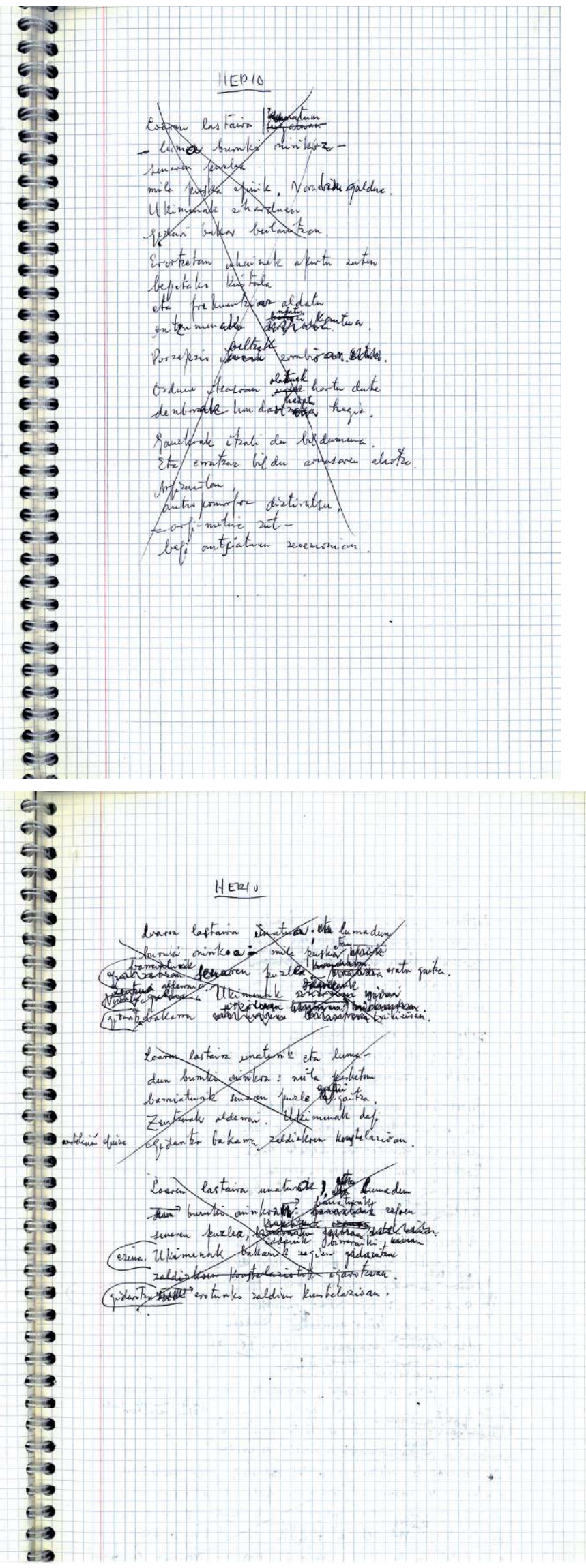

Fig. 7a et 7b : Poème Herio-Muerte [Mort]. Dossier 3.

Première phase (fig. 7a), texte en basque à droite et texte autotraduit en espagnol à gauche, puis barrés. Deuxième phase (fig. 7b), seul le texte basque est repris à droite

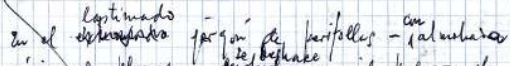

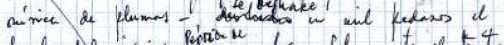

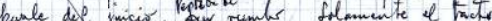

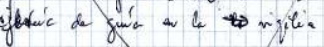

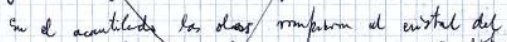

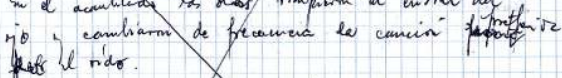

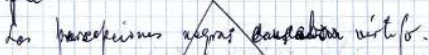

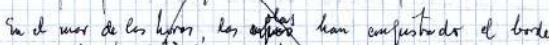

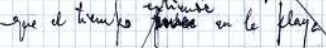

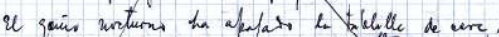

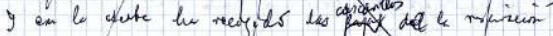

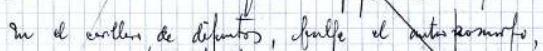

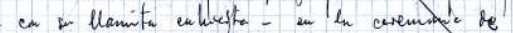
mirades ansiostas.

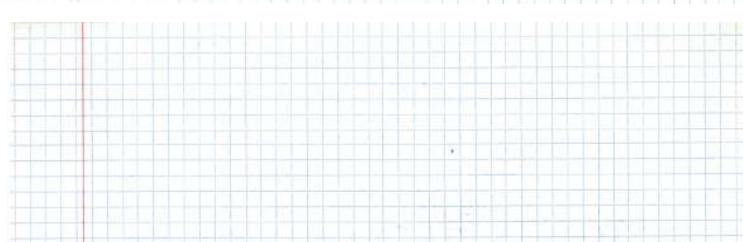


Lastaina unatua, lumadum burkei

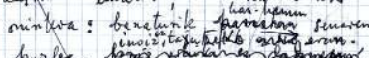

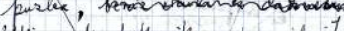

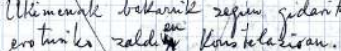
Peleck sistem dhot b busitale.

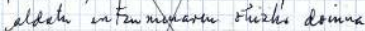

Prrakzionte elamer suder st taliak ito ordum itraboon arkinativi iada

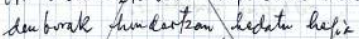
Hurtilife orim luma te cesereza!

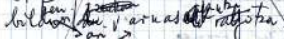

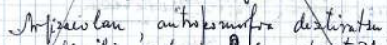

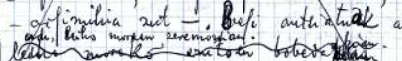

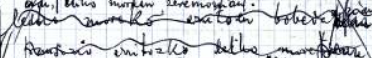

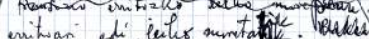

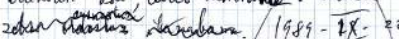

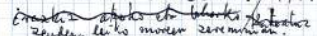

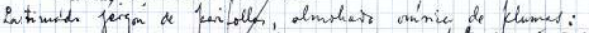

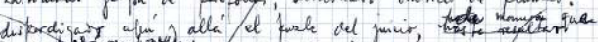

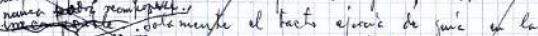

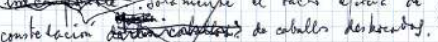

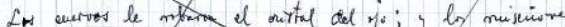

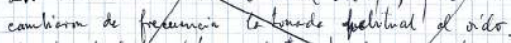

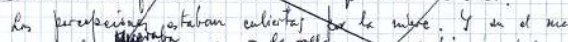

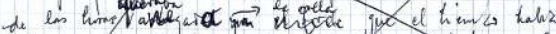

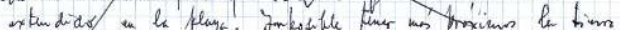

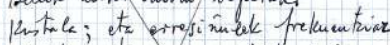

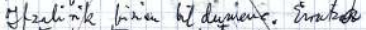
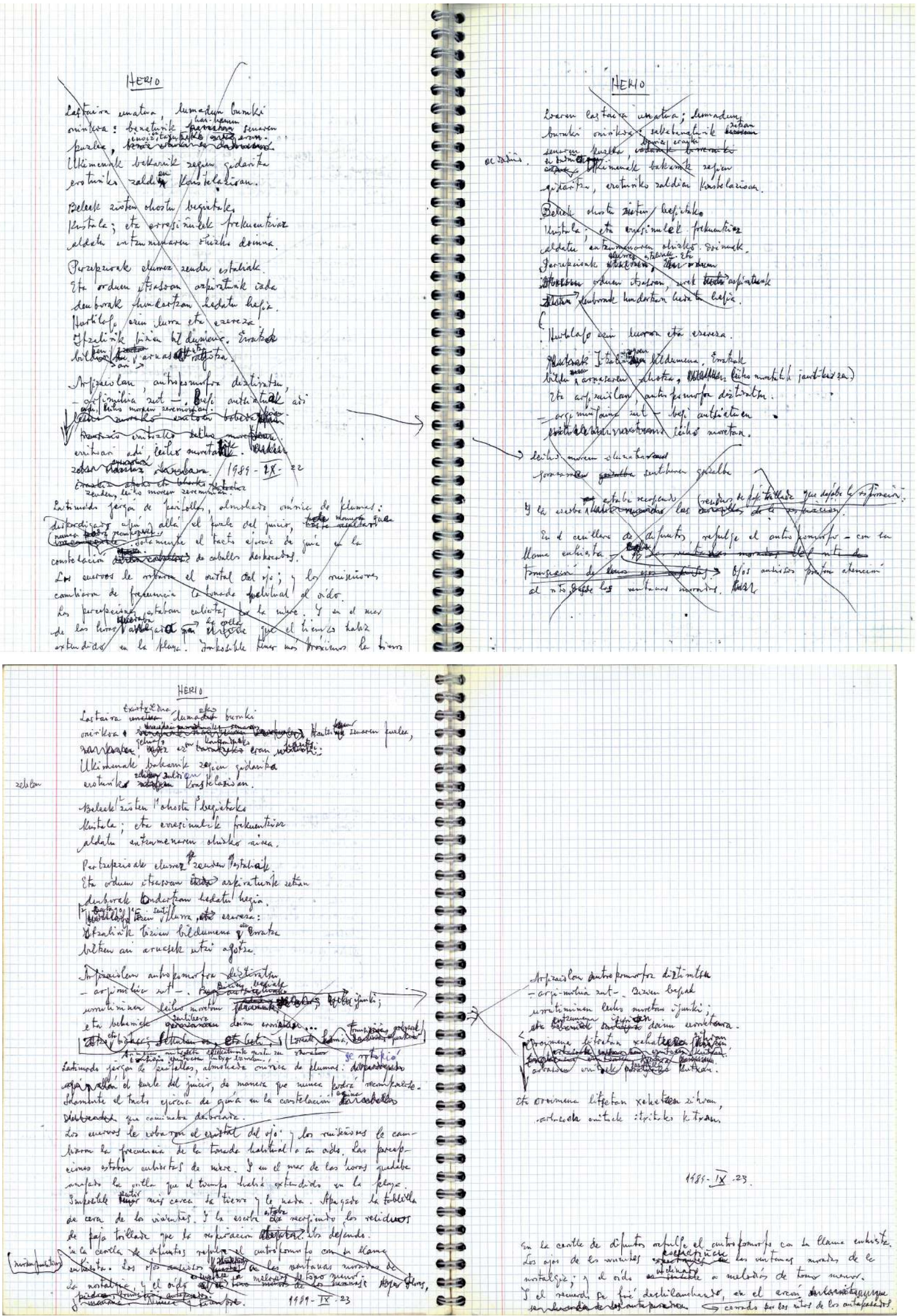

Fig. 7c et 7d : Poème Herio-Muerte [Mort]. Dossier 3 

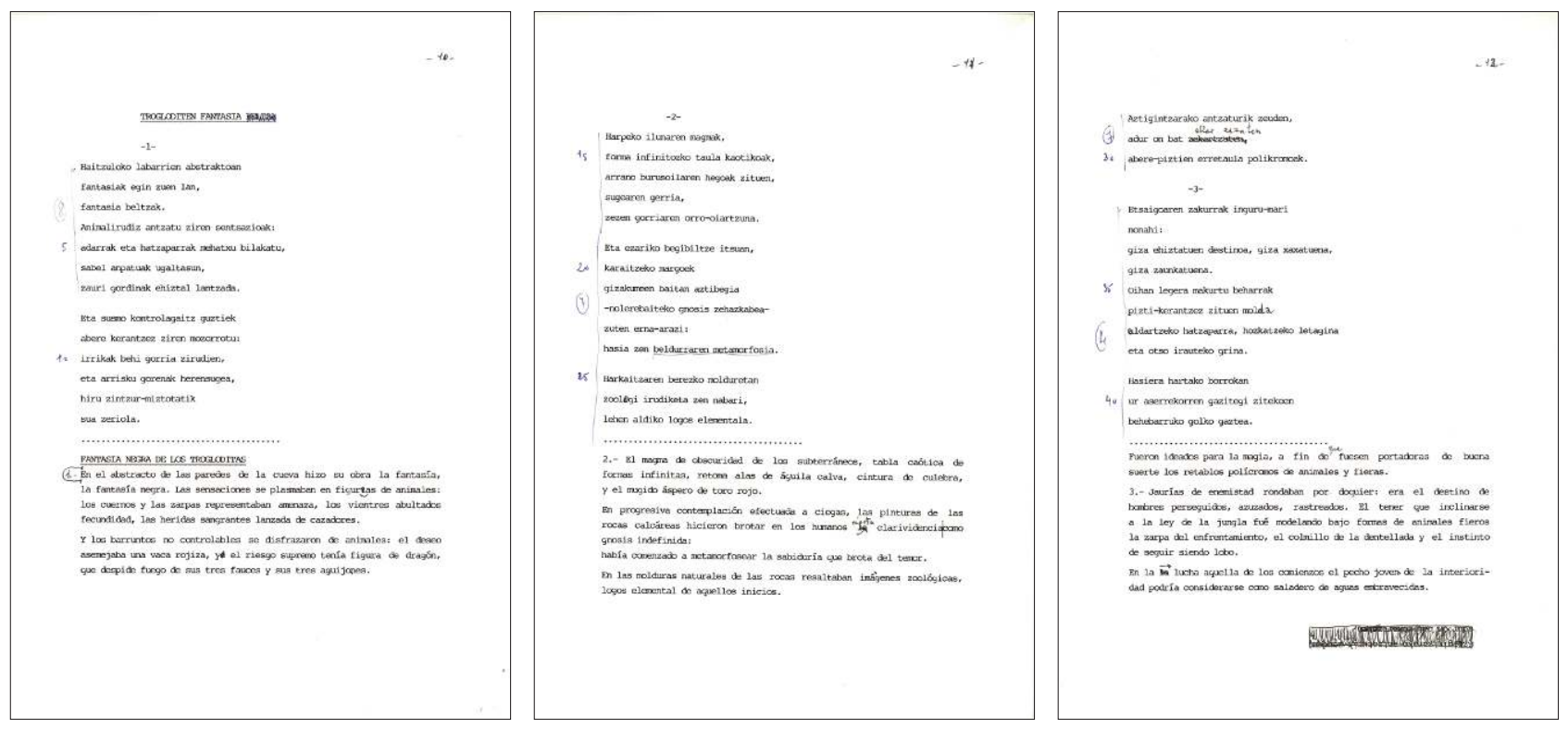

Fig. 8a, 8b et 8c : Trogloditoen fantasia beltza - Fantasía negra de los trogloditos [Fantaisie noire des Troglodites] Dossier 1 «Lehen hurbilketak (1982-1987)» [Premières approches (1982-1987)]
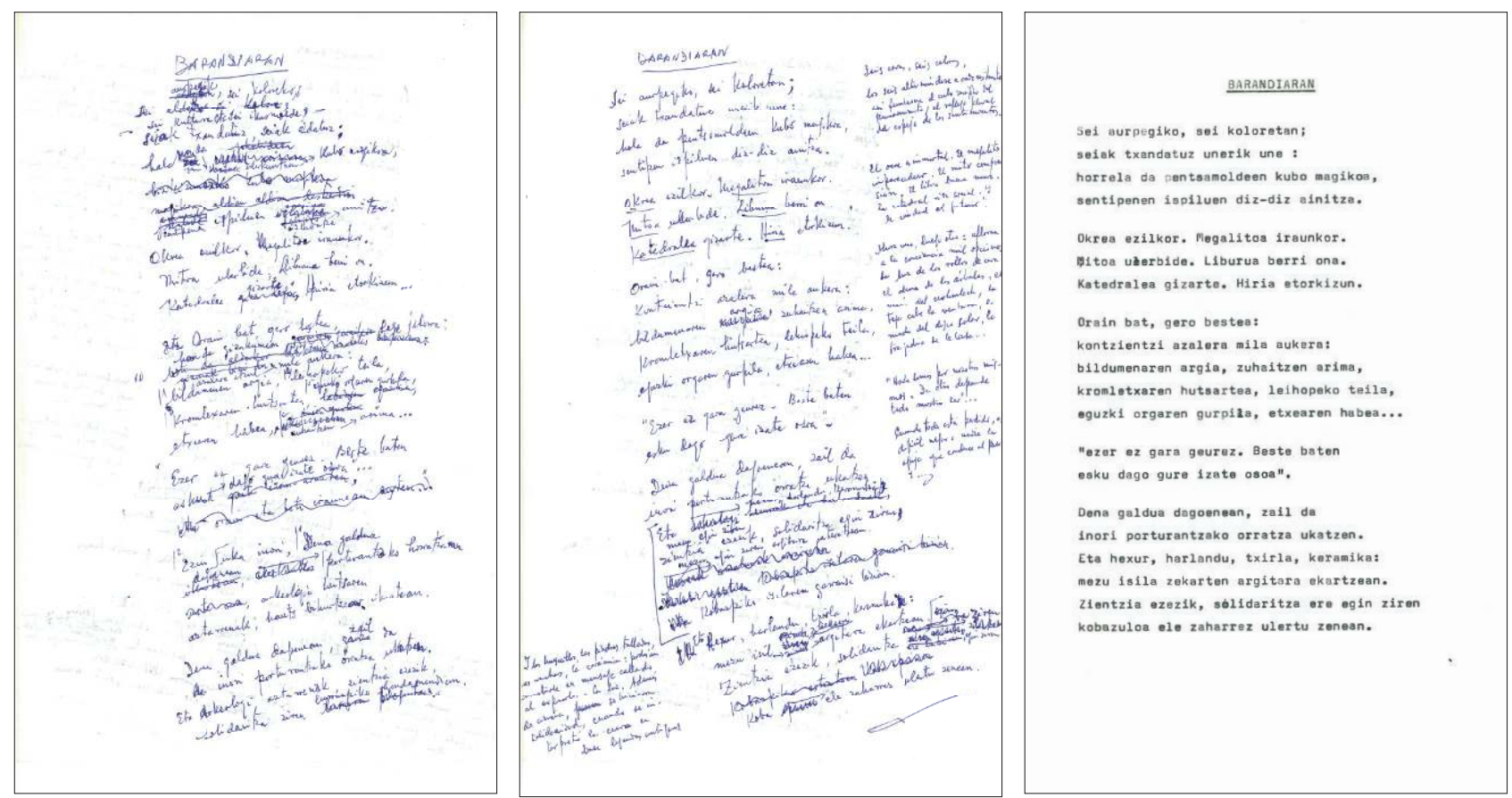

Fig. $9 \mathrm{a}, 9 \mathrm{~b}$ et $9 \mathrm{c}:$ Les trois campagnes d'écriture du poème Barandiaran.

Dossier 2 intitulé «Lehen zirriborroa...» [Premiers brouillons], «Uztaren azkenetan bukatua» [Terminé fin 1989] 
Lors d'une troisième campagne d'écriture intervenue le 31 août 1989, le poème est repris en langue basque sur la page de droite et autotraduit en espagnol sur la page de gauche, deux vers posant problème sont repris en langue basque, autotraduits puis repris en basque et à nouveau autotraduits, et ainsi plusieurs fois. Trois directions se dessinent : de haut en bas puis de droite (basque) à gauche (espagnol) et de gauche (espagnol) à droite (basque) et à nouveau à gauche (basque) le texte est retravaillé.

Enfin, lors de la quatrième et dernière campagne d'écriture, une mise au propre s'effectue. On se rend compte que les versions en langue basque datent des 1er et 2 septembre 1989 (à droite), tandis que l'autotraduction en espagnol est stoppée au 1er septembre (à gauche). À l'évidence ici comme ailleurs, la version en espagnol a remis en mouvement le texte en basque puisqu'il est repris le 2 septembre (sans autotraduction) (fig. 6d).

Même si le poète ne semble pas viser la publication en langue espagnole, cela n'empêche pas qu'il passe du temps à affiner la version en cette langue. La version en espagnol joue le rôle d'une sorte de mise au net qui constitue un état textuel supplémentaire. Tout cela marque à la fois la primauté de l'écriture en basque et, par conséquent, le caractère consécutif 28 de ce processus de création bilingue.

Observons à présent quelques exemples de ce processus autotraductif.

Le maniement matériel du cahier à spirales, utilisé grandouvert ou pas, rend plus manifeste encore le caractère consécutif de l'autotraduction. La spatialité peut s'en trouver bouleversée ainsi que le montrent les diverses campagnes d'écriture du poème Herio-Muerte [Mort] (fig. 7a, 7b, 7c et 7d).

Lors de la troisième phase (fig. $7 \mathrm{c}$ ), le texte en basque et le texte autotraduit franchissent la frontière matérialisée par la spirale (flèche directionnelle) et se retrouvent aussi bien à droite qu'à gauche (l'une des versions en basque porte la date du 22 septembre 1989), puis toutes les tentatives sont barrées. Ensuite, le texte en basque se retrouve sur la gauche (fig. 7d), il est traduit en espagnol en dessous, les parties barrées en basque et en espagnol passent sur la page en face (flèche directionnelle du texte en basque barré vers la reprise) et sont retravaillées sur la droite; la date du 29 septembre 1989 figure sous le fragment en basque retravaillé à droite.
Le caractère consécutif de l'autotraduction en espagnol s'observe également dès les premières campagnes de la phase rédactionnelle (Dossier 1). Il en est ainsi pour le poème Trogloditoen fantasia beltza - Fantasía negra de los trogloditos [Fantaisie noire des Troglodites] (fig. 8a, $8 \mathrm{~b}$ et $\left.8 \mathrm{c}^{29}\right)$.

On voit que l'adjectif en basque beltza [noire] est biffé, alors que l'adjectif en espagnol negra [noire], qui lui correspond, ne l'est pas. Probablement, une fois le texte traduit en espagnol, l'écrivain est revenu sur le texte basque mais non pas sur sa version espagnole. Mais on observe également que Lekuona effectue des allers et retours dans le même texte dactylographié, en travaillant ensuite de manière indépendante le texte en espagnol : par exemple, le terme basque azpilduretan [dans les ourlets] est d'abord traduit par (en los) entresijos [dans les coulisses], puis entresijos est biffé et remplacé par repliegos [replis], comme si Juan Mari Lekuona recherchait le rendu le plus exact possible du texte basque en espagnol. Il fera de même pour plusieurs autres poèmes autotraduits. Cela confirme bien notre hypothèse que le texte en espagnol fonctionne vraiment comme une nouvelle version du texte original en basque.

L'exemple du poème Barandiaran 30 montre bien le rôle que la langue espagnole joue dans sa création. Les documents disponibles indiquent qu'il y a eu au moins trois campagnes d'écriture (fig. 9a, 9b et 9c) : 1) une première version manuscrite en basque avec de nombreuses ratures (fig. 9a); 2) une seconde version qui est une mise au net recorrigée et autotraduite en espagnol dans les marges de cette même page (fig. 9b). On peut y remarquer que Lekuona traduit vers par vers, en essayant de rendre la rythmique du texte; 3 ) une dernière version dactylographiée monolingue en basque (fig. 9c) qui ne correspond pas exactement au manuscrit corrigé avec autotraduction, mais montre la finalité poursuivie (le texte sera publié uniquement en basque).

28. Sur la distinction entre l'écriture consécutive et simultanée, voir Olga Anokhina et Emilio Sciarrino ici même.

29. Dans la partie autotraduite en espagnol le titre est suivi d'une note entre parenthèses en espagnol bien visible : «Traducción para bilingües » [Traduction pour bilingues]. Une autre note indique qu' «une version proche de celle-ci fut lue à Tolosa le 24 août 1986 ».

30. Du nom de l'abbé Barandiaran, préhistorien et ethnologue. 
Au vu de la différence importante entre l'état 2 (fig. 9b) et 3 (fig. 9c), on peut supposer que les étapes intermédiaires (texte bilingue dactylographié) manquent. Ce poème continuera à être corrigé et retravaillé uniquement en basque jusqu'à la dernière version telle qu'elle a été donnée à l'éditeur.

\section{Conclusion}

Mimodrames et Icônes n'est pas un ouvrage bilingue autotraduit, il serait donc difficile de parler à son propos de «communication dédoublée »31. Divers éléments, comme la spatialité de la mise en page, la datation, le caractère consécutif ou non des corrections, les oublis de la traduction, etc. montrent qu'il s'agit ici d'autotraduction ou d'écriture consécutive, même si, à certains moments, il y a des interactions entre les deux langues.

Au fil de l'avancement du projet d'écriture, la version en espagnol, qui a pu revêtir diverses formes, retravaillée, interrompue momentanément, s'arrête définitivement une fois que la phase rédactionnelle en basque est bien avancée.
Il est important de souligner que le poète est resté très discret sur son usage de la langue espagnole peut-être en raison du contexte de constitution de la littérature basque et de son émancipation du système linguistique et littéraire espagnol. Enfin, il a pu vouloir privilégier la publication en langue basque (sans autotraduction) pour des raisons esthétiques :

La théorisation du thème (de Mimodrames et Icônes) aurait pu être écrite dans n'importe quelle langue. Mais ces mimodrames et ces images ont un nom précis et riche en langue basque, avec mille nuances qui ne se trouveraient en aucune autre langue 32 .

31. $C f$. Rainier Grutman, «Francophonie et autotraduction», dans Paola Puccini (dir.), Inter-francophonies, $\mathrm{n}^{\circ} 6$, «Regards croisés autour de l'autotraduction, 2015, p. 8. En ligne : <www.interfrancophonies.org > (consulté en décembre 2017).

32.«Poetika baten bilakabidea eta azpiko hariak» [Devenir d'une poétique et trame secrète] (1996), dans Ibilaldia-Itinerario [Itinéraire], op. cit., p. 43.

Aurelia ARCOCHA-SCARCia est professeur à l'université Bordeaux Montaigne. Ses recherches portent sur le processus de création et les phénomènes de plurilinguisme dans la littérature contemporaine d'expression basque. Elle travaille également sur les livres imprimés et les archives des fonds basques (XVIe-XIXe). Elle fait partie de l'équipe Multilinguisme, Traduction, Création de l'ITEM et a co-dirigé l'ouvrage En el taller del escritor. Génesis textual y edición de textos (2010). 


\section{Les deux langues en clair-obscur dans Mimodrames et lcônes de Juan Mari Lekuona}

Les brouillons du dernier ouvrage publié en langue basque par le poète Juan Mari Lekuona (1927-2005), Mimodramak eta Ikonoak (Mimodrames et Icônes) révèlent la présence d'une deuxième langue, l'espagnol, qui agit comme un miroir dans le processus créatif. Cette pratique autotraductive concerne la phase rédactionnelle de la création, après une première maturation en langue basque. Dans les dernières étapes du processus d'écriture, la seule langue utilisée sera le basque, et Mimodrames et Icônes sera édité uniquement en langue basque en 1990. Ni le lecteur ni le critique ne peuvent en soupçonner l'épaisseur bilingue s'ils n'ont pas accès aux brouillons. Pourtant, la pratique autotraductive permet à Lekuona d'augmenter le champ chromatique de la langue de création destinée à la publication. Pourquoi le poète, lui-même traducteur, n'a-t-il pas publié ses autotraductions ? Pourquoi n'en a-t-il pas parlé ? Cet article aborde les particularités d'une pratique poétique autotraductive tenue secrète, chez l'un des plus importants poètes contemporains du Pays basque péninsulaire, en Espagne.

The drafts of Juan Marie Lekuona's last book published in Basque, Momodramak eta Ikonoak, reveal the presence of a second language, Spanish, which acts as a mirror in the creative process. This self-translation practice appears in the editorial stage, after a first maturing period in Basque. In the last stage of the writing process, Basque is the only language used, and until 1990 Momodramak eta Ikonoak was published only in Basque. Without seeing these drafts, neither the reader nor the critic can even guess at the bilingual depth of the book. Yet, the practice of autotranslation allowed Lekuona to increase the chromatic field of the language destined for publication. Why did the poet, a translator himself, not publish his self-translations? Why did he never speak of them? This article approaches the characteristics of a secret practice of self-translation in one of the most important contemporary poets of the Basque Country in Spain.

Die Brouillons des letzten auf Baskisch verfassten Werkes des Dichters Juan Mari Lekuona (1927-2005), Mimodramak eta Ikonoak (Mimodrama und Ikonen) zeigen die Anwesenheit einer zweiten Sprache, nämlich des Spanischen, die wie ein Spiegel im kreativen Prozess fungiert. Diese Praxis des Sich-SelbstÜbersetzens betrifft die redaktionelle Phase des Schaffens, die einem ersten Entwurf in nur baskischer Sprache folgt. In den letzten Etappen des Schreibprozesses ist das Baskische wieder die einzige verwendete Sprache, und Mimodrames et Icônes wird im Jahre 1990 nur in Baskisch herausgegeben. Weder der Leser noch der Kritiker kann die bilinguale Tiefe darin vermuten, wenn sie nicht zu den Brouillons Zugang haben. Die Praxis des Sich-SelbstÜbersetzens erlaubt Lekuona jedoch eine Erweiterung der Farbpalette jener Sprache, in der er publizieren will. Warum hat der Dichter, der selbst Übersetzer ist, nicht seine eigenen Übersetzungen veröffentlicht? Warum hat er darüber nicht gesprochen? Der vorliegende Artikel nähert sich den Besonderheiten einer poetischen Praxis des Sich-Selbst-Übersetzens, die geheim gehalten wurde, bei einem der wichtigsten zeitgenössischen Dichter des spanischen Baskenlands.
Los borradores de la última obra publicada en lengua vasca por el poeta Juan María Lekuona (1927-2005), Mimodramak eta Ikonoak (Mimodramas e Íconos), revelan la presencia de un segundo idioma, el español, que actúa como un espejo en el proceso creativo. Esta práctica autotraductiva se ejerce durante la fase redaccional de la creación, después de una maduración en lengua vasca. En las últimas etapas del proceso de escritura, el único idioma utilizado será el vasco, y Mimodramas e Íconos será editado únicamente en ese idioma en 1990. Es imposible para los lectores y los críticos imaginar este espesor bilingüe si no han tenido acceso a los borradores. Sin embargo, la práctica autotraductiva permite a Lekuona aumentar el campo cromático de la lengua de creación destinada a la publicación. ¿Por qué el poeta, siendo traductor él mismo, no ha publicado sus autotraducciones? ¿Por qué no las ha mencionado? Esta artículo trata de las particularidades de una práctica poética autotraductiva mantenida secreta por uno de los poetas contemporáneos más importantes del País Vasco peninsular, en España.

Os rascunhos do último livro publicado em língua basca pelo poeta Juan Mari Lekuona (1927-2005), Mimodramak eta Ikonoak (Mimodramas e ícones) revelam a presença de uma segunda língua, o espanhol, que atua como espelho no processo criativo. Esta prática de autotradução insere-se na fase criativa de redacção, após uma maturação inicial em língua basca. Na fase final do processo de escrita, a única língua usada será o basco, e nele somente será publicada a obra em 1990. Nem o leitor nem o crítico podem suspeitar a espessura bilingue se não tiverem acesso aos rascunhos. No entanto, a prática de autotradução confere a Lekuona a possibilidade de incrementar o campo cromático da linguagem da criação destinada a publicação. Por que motivo o poeta, que é ele mesmo tradutor, não publicou as suas autotraduções? Porque não revelou a sua existência? Este artigo aborda as peculiaridades de uma prática poética de autotradução automática mantida secreta, naa obra de um importante poeta basco contemporâneo.

Le bozze dell'ultima opera pubblicata in lingua basca dal poeta Juan Mari Lekuona (1927-2005), Mimodramak eta Ikonoak (Mimodrammi e Icone) rivelano la presenza di una seconda lingua, lo spagnolo, che agisce come uno specchio durante il processo creativo. Questa pratica autotraduttiva viene impiegata nella fase redazionale della creazione, dopo una prima maturazione in lingua basca. Nelle ultime fasi del processo di scrittura, l'unica lingua utilizzata sarà il basco e Mimodrammi e Icone verrà pubblicato unicamente in lingua basca nel 1990. Né il lettore né il critico possono immaginare lo spessore bilingue senza avere accesso alle bozze. Eppure, la pratica autotraduttiva permette a Lekuona di aumentare il campo cromatico della lingua di creazione destinata alla pubblicazione. Perché il poeta, che è anche traduttore, non ha pubblicato le sue autotraduzioni? Perché non ne ha parlato? L'articolo esamina le particolarità di una pratica poetica autotraduttiva tenuta segreta, in uno dei più importanti poeti contemporanei dei Paesi Baschi. 